\title{
Self-Association of Aromatic Oligoamide Foldamers into Dou- ble Helices in Water
}

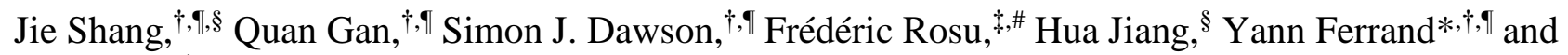 \\ Ivan $\mathrm{Huc}^{*, \dagger}$, Il \\ $\dagger$ Univ. Bordeaux, CBMN, UMR 5248, Institut Européen de Chimie Biologie, 2 rue Robert Escarpit 33607 Pessac, France. \\ II CNRS, CBMN, UMR 5248, 33607 Pessac, France. \\ $\S$ Beijing National Laboratory for Molecular Sciences, CAS Key Laboratory of Photochemistry, Institute of Chemistry, Chi- \\ nese Academy of Sciences Beijing 100190, China. \\ † Univ. Bordeaux, UMS 3033/US 001, IECB, 2 rue Robert Escarpit 33607 Pessac, France. \\ \# CNRS, IECB, UMS 3033, 33607 Pessac, France.
}

Supporting Information Placeholder

$2 \times$
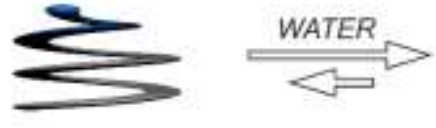

Single Helix

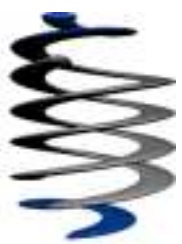

Double Helix

\begin{abstract}
Heteromeric oligoamide foldamers composed of 8-amino-2-quinolinecarboxylic acid and 7-amino-8-fluoro-2-quinolinecarboxylic acid bearing cationic water solubilizing side chains were prepared using solid phase synthesis (SPS). The sequence was designed to adopt a single or a double helical motif depending on the nature of the solvent, DMSO or water, respectively. Selfassociation was demonstrated by NMR and mass spectrometry. Dimerization in water was found to be much higher than observed previously in organic solvents for analogous oligoamide sequences.
\end{abstract}

The self-aggregation of organic oligomeric or polymeric molecular strands into multiple helices is a commonly observed phenomenon in nature. ${ }^{1}$ Chemists have mimicked this process in a variety of systems and shown that artificial organic oligomers are also able to associate into multistranded structures using original recognition motifs (a vast array of helicates involving metal coordination also exist and is not being considered here). As prime examples, multiple helical structures have been evidenced in oligoamides comprised of pyridine, ${ }^{2}$ chloro- or fluoro-aromatic ${ }^{3}$ or naphthyridine ${ }^{4}$ rings; in oligo-resorcinols; ${ }^{5}$ in ethynylhelicene oligomers ${ }^{6}$ in meta-terphenyl backbone oligomers exploiting amidinium-carboxylate salt bridges ${ }^{7}$ and in alternate sequences of aromatic hydrogen-bond donors and acceptors. ${ }^{8}$ However, most of these multi-stranded structures only form in organic solvents. The transposition of such architectures into water is far less common and no family of organic double helices has been reported to form in essentially any solvent. To our knowledge, apart from the notable exception of Yashima's oligoresorcinols, ${ }^{5,9}$ no other example of double helix association in water based on non-natural motifs has been described so far. In this context, worth mentioning also are Iverson's aedamers which represent an original class of aromatic oligomers able to fold and aggregate as discrete hetero-duplexes in water, though they do not possess a helical architecture. ${ }^{10}$ The latter two systems both rely extensively on aromatic-aromatic stacking interactions between complementary oligomeric strands. In line with this background, we now report the association of quinoline oligoamide strands into remarkably stable anti-parallel helical duplexes in water.

The prospect to associate helical aromatic oligoamide foldamers in water potentially brings concerns about their solubility and about their ability to remain folded in this medium. ${ }^{11}$ However, it has recently been shown that, in spite of their aromatic nature, they can be easily solubilized in water provided polar side-chains diverge from the structure. ${ }^{12}$ Moreover, it was demonstrated that their conformational stability can in fact be dramatically enhanced in this solvent, presumably due to the strength of hydrophobic effects. ${ }^{13}$ We thus speculated that the introduction of ornithine-like side chains at the periphery of a sequence prone to double helix formation in organic solvents might lead to self-association in water. Inspired by an earlier design, ${ }^{14}$ we targeted oligoamide sequences comprised of both single and double helical segments so as to promote exclusively antiparallel duplex formation (Figure 1). Specifically, a quinoline trimer $\left(\mathbf{Q}_{3}\right)$ at the $\mathrm{C}$-terminus codes for a short single helical segment that has no propensity to associate. In contrast, the fluoroquinoline segment $\left(\mathbf{Q}^{\mathbf{F}}\right)_{\mathrm{n}}$ at the $\mathrm{N}$-terminus was expected to self-associate, as previously shown in organic solvents, but only in an anti-parallel fashion. ${ }^{14}$ This is because the $\mathbf{Q}_{3}$ segment serves to prevent parallel duplex formation which would require 
severe steric hindrance or disfavored association to occur at one end. Energy minimized molecular models indicated a helix pitch of $3.5 \AA$ in single helical monomeric 1 whereas the models showed the helix pitch to be 3.5 and $7 \AA$ for the $\mathbf{Q}_{\mathbf{3}}$ and $\mathbf{Q}^{\mathbf{F}} \mathbf{4}$ segments of $(\mathbf{1})_{2}$, respectively (Figure 2 ). In the modelled duplex, interstrand contacts consist mainly of face-to-face aromatic stacking of the $\mathbf{Q}^{\mathbf{F}}$ units. The aromatic surface exposed to solvent was found to be strongly reduced in the duplex compared to the single helix, which potentially represents a driving force in a solvophobic context (see supporting information). Specifically, the calculated solvent accessible surface for double helical $(\mathbf{2})_{2}$ and single helical 2 were found to be $1655 \AA^{2}$ and $1021 \AA^{2}$, respectively. Thus, one can estimate that a double helix $(2)_{2}$ exposes to the solvent only about $80 \%$ of the surface exposed by two individual single helices.

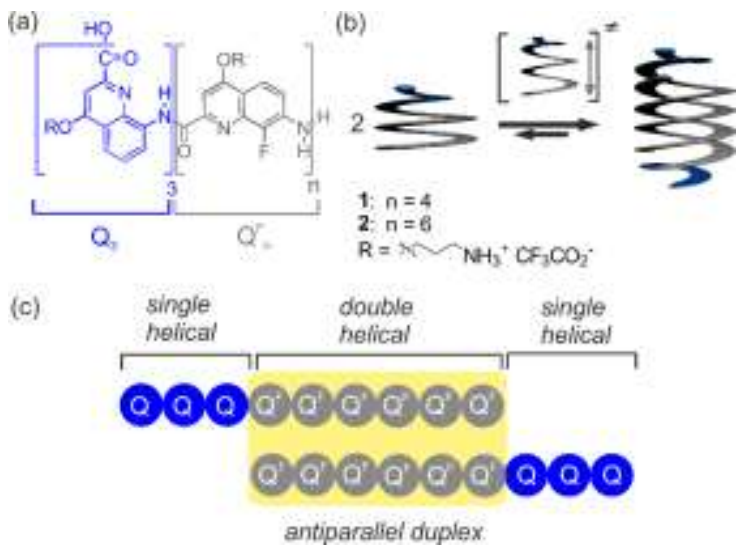

Figure 1. (a) Formula of $\mathbf{1}$ and 2; (b) Schematic representation of a single-helix double-helix equilibrium requiring spring-like extension; (c) Simplified representation of double helix (2) 2 . Quinolines are denoted in blue whereas Fluoroquinolines are shown in light grey.

We opted for the solid phase synthesis (SPS) of oligomers 1 and 2. SPS brings the benefits of speed and, in the present instance, elongation from the surface of resin beads also advantageously prevents duplex formation from occurring during synthetic steps. Indeed, our experience is that the formation of stable duplexes in solution seriously hampers strand elongation reaction. ${ }^{15}$ The TFA-labile Wang resin was selected as it offered the possibility to synthesize oligomers using an Fmoc-type strategy. ${ }^{15 a}$ For this purpose, we needed to prepare both the previously described Fmoc-protected 8-amino-2-quinolinecarboxylic acid monomer bearing a Boc protected aminopropoxy side chain 3 and the new Fmoc-protected 7-amino-8-fluoro-2-quinolinecarboxylic acid monomer $\mathbf{4}$, the synthesis of which is shown in Scheme 1. Starting from quinolone 5, Boc protected aminopropanol was introduced under Mitsunobu conditions (69\%). The nitro group was then reduced via catalytic hydrogenation with $\mathrm{Pd} / \mathrm{C}$. The resulting amine was protected using Fmoc- $\mathrm{Cl}$ and the Fmoc quinoline acid derivative $\mathbf{4}$ subsequently obtained in moderate yield $(52 \%)$ through the cleavage of its methyl ester using a LiI mediated nucleophilic dealkylation. High purity of this material is essential to successful SPS.

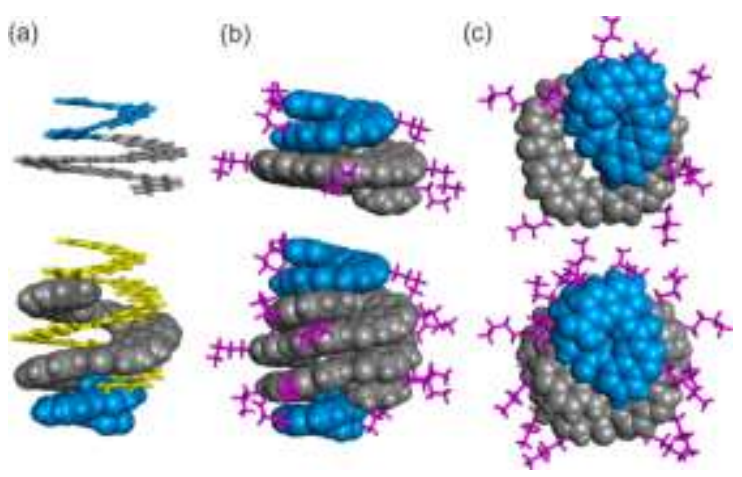

Figure 2. Side (a,b) and top (c) views of energy-minimized models of single helical $\mathbf{2}$ and double helical (2)2. Aromatic backbones are color coded in blue (quinoline) and grey (fluoroquinoline). In (a), one strand of $(2)_{2}$ is shown in a yellow tube representation. Side chains were omitted for clarity. In (b) and (c), 2 and (2) 2 are shown as CPK models. Side chains are shown as purple tubes.

Scheme 1. Formulas and synthesis of Fmoc-quinoline derivatives 3 and $\mathbf{4}$.

(a)

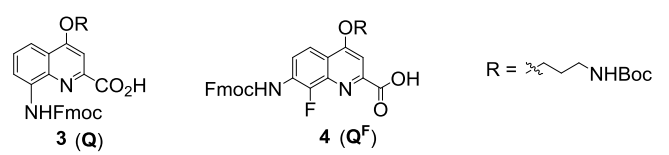

(b)

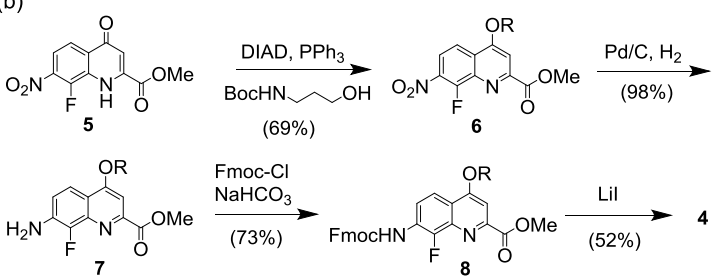

Consistent with the structures of $\mathbf{1}$ and $\mathbf{2}$ and to exploit our previous experience with SPS of quinoline oligoamides, ${ }^{15 \mathrm{a}}$ it was decided to elongate the oligomer from a quinoline $\mathrm{C}$-terminus $(\mathrm{Q})$ to a fluoroquinoline $\mathrm{N}$-terminus $\left(\mathrm{Q}^{\mathrm{F}}\right)$ (Scheme 2). However, the reverse sequence where synthesis would start with the $\left(Q^{F}\right)_{n}$ segment and the $N$-terminus would be at the end of the $Q_{3}$ segment may have also been envisaged. Quinoline acid monomer 3 was introduced onto low loading $\left(0.38 \mathrm{mmol} \mathrm{g}^{-1}\right)$ Wang bromide resin in the presence of cesium iodide under microwave heating. Fmoc deprotection was carried out at room temperature by treatment with piperidine, and the second Q unit incorporated via the acid chloride 3-Cl under microwave heating (Scheme 2). Another deprotection-coupling sequence was performed to add a third quinoline to the resin. Fluoroquinoline monomers were then introduced successively using a similar deprotection-coupling protocol via the acid chloride $4-\mathbf{C l}$ (Scheme 2). Concomitant side chain deprotection and cleavage from the resin was carried out in 95:2.5:2.5 TFA $/ i \mathrm{Pr}_{3} \mathrm{SiH} / \mathrm{H}_{2} \mathrm{O}$ ( $\mathrm{vol} / \mathrm{vol} / \mathrm{vol})$. The water solu-ble oligomers 1 and $\mathbf{2}$ were then purified by reversed-phase HPLC prior to physico-chemical studies. Purification was difficult in the case of $\mathbf{2}$.

Scheme 2. Solid phase synthesis (SPS) of $\mathbf{1}$ and $\mathbf{2}$. 

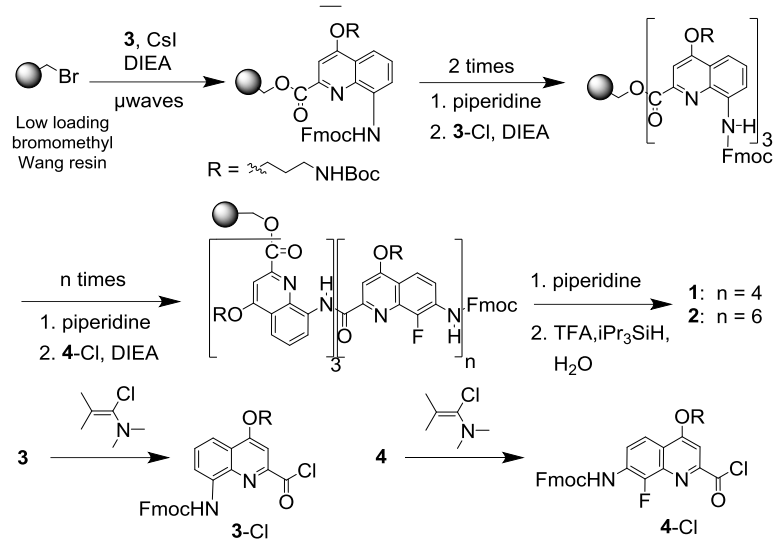

Oligomers $\mathbf{1}$ and $\mathbf{2}$ were designed to be soluble both in polar organic solvents and pure (i.e. non-buffered) water. The aggregation behavior of 1 was initially studied in $d_{6}$-DMSO, a solvent known to disfavor the aggregation of aromatic oligoamides. In this solvent, a single set of signals was observed at $2 \mathrm{mM}$ and assigned to a single helix (Figure 3a). Upon increasing the water content, a new set of signals appeared at lower field (Figure 3b$\mathrm{d}$, green) which is characteristic of duplex formation. ${ }^{2}$ This observation was consistent with a slow exchange between $\mathbf{1}$ and $(\mathbf{1})_{2}$ on the NMR time scale. Similar upfield shifts of amide signals have been observed in organic solvents and represent a typical signature of ring current effects arising from extended intermolecular $\pi-\pi$ stacking within double helical aggregates. Although a full structural elucidation of $(\mathbf{1})_{2}$ was not carried out, its assignment to a double helical structure such as that shown in Figure 2 could be inferred by analogy to related sequences in organic solvents. ${ }^{14}$ Importantly, in agreement with our design to favor antiparallel duplexes, a single set of signals was observed for the aggregate. In addition, the multiplicity of these signals indicated that the molecules of $\mathbf{1}$ involved in the aggregate are magnetically equivalent. Given the previous characterization of related anti-parallel duplexes in organic solvents, ${ }^{3 a}$ and in agreement with molecular models (see below) these data make it very likely that an anti-parallel duplex also prevails in water.

To assess its dimerization strength, 1 was dissolved in $d_{6^{-}}$ DMSO containing varying proportions of water. Upon increasing the water content from 0 to $30 \% \mathrm{vol} / \mathrm{vol}$, the signals of single helical 1 were progressively replaced by peaks corresponding to the double helix $(\mathbf{1})_{2}$. The dimerization constant could be calculated through the integration of the signals of the two species at a total concentration of $2 \mathrm{mM}$ and was found to be $10^{3} \mathrm{~L}$ $\mathrm{mol}^{-1}$ in a $\mathrm{H}_{2} \mathrm{O}: d_{6}$-DMSO 20:80 (vol/vol) mixture. A slight increase of this ratio (26:74 vol/vol) led to an increase of the $K_{\text {dim }}$ by two orders of magnitude $\left(10^{5} \mathrm{~L} \mathrm{~mol}^{-1}\right)$. Above $30 \%$ of water in DMSO oligomer 1 proved to exist solely as a double helix as judged by the unique set of resonances by ${ }^{1} \mathrm{H}$ NMR (Figure $3 \mathrm{~d}$ ). The dimerization constant of $\mathbf{1}$ in pure water was too high to be measured by NMR. Dimerization was also observed upon adding a $20 \mathrm{mM}$ aqueous solution of $\mathrm{D}_{11}$-Tris buffer at $\mathrm{pH}$ 6.2. In comparison, an organic soluble $\left(\mathbf{Q}^{\mathbf{F}}\right)_{4}$ analogue was previously shown to only weakly aggregate in chloroform. ${ }^{3 a}$ Double helix formation of aromatic oligoamides thus appears to be strongly enhanced in water, possibly due to the same factors which also enhances single helix stability, ${ }^{13}$ i.e. hydrophobic effects.
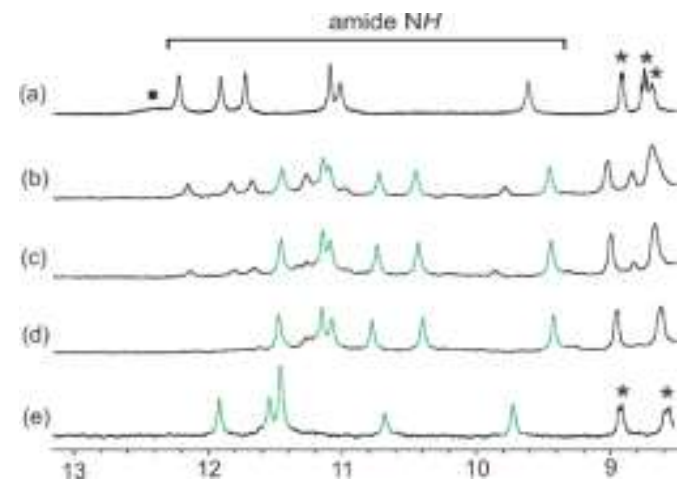

Figure 3. Excerpts of the ${ }^{1} \mathrm{H}$ NMR $(400 \mathrm{MHz})$ titration at $298 \mathrm{~K}$ of $1(2 \mathrm{mM})$ in $\mathrm{d}_{6}-\mathrm{DMSO} / \mathrm{H}_{2} \mathrm{O}$ mixtures in the following vol/vol ratios: (a) 100:0; (b) 80:20; (c) 76:24; (d) 70:30. (e) ${ }^{1} \mathrm{H}$ NMR (400 MHz) spectrum of $(\mathbf{1})_{2}$ in water $\left(\mathrm{H}_{2} \mathrm{O}: \mathrm{D}_{2} \mathrm{O} 90: 10 \mathrm{vol} / \mathrm{vol}\right)$. Black and green colored signals stand for single and double helix, respectively. Some aromatic resonances are denoted with stars. The signal of the carboxylic acid is marked with a black square.

The self-association of longer oligomer 2 was investigated by electrospray mass spectrometry (ESI-MS). This method allows to unambiguously determine the stoichiometry of supramolecular aggregates. ${ }^{16}$ ESI-MS measurements of $\mathbf{2}$ were performed in water. The oligomer appeared as multiple positively charged species, corresponding to the mass of the single and double helices (Figure 4a, green helices). Monomeric 2 was detected at $m / z=772.6,1158.4(3+$ and $2+$ species, respectively) and the double helix $(2)_{2}$ was detected at $m / z=1158.4,1544.2$, 2315.8 (4+, 3+ and 2+ species, respectively). A closer examination of the mass spectrum allowed us to identify a molecular adduct of $\mathbf{2}$, the mass of which was larger than that of $\mathbf{2}$ by 95.97 mass units (red helices) This adducts presumably corresponds to a trifluoroacetylation $\left(2-\mathrm{COCF}_{3}\right)$ which likely occurs at the $\mathrm{N}$-terminal $\mathrm{Q}^{\mathrm{F}}$ aromatic amine function during TFA-mediated resin cleavage as a result of incomplete protonation of this amine. As a consequence of the presence of this species, the mass spectrum also shows peaks corresponding to a cross-hybrid between $\mathbf{2}$ and $\mathbf{2}-\mathrm{COCF}_{3}$ a species that can be regarded as additional evidence of the strong aggregation propensity of these oligomers into duplexes in water.

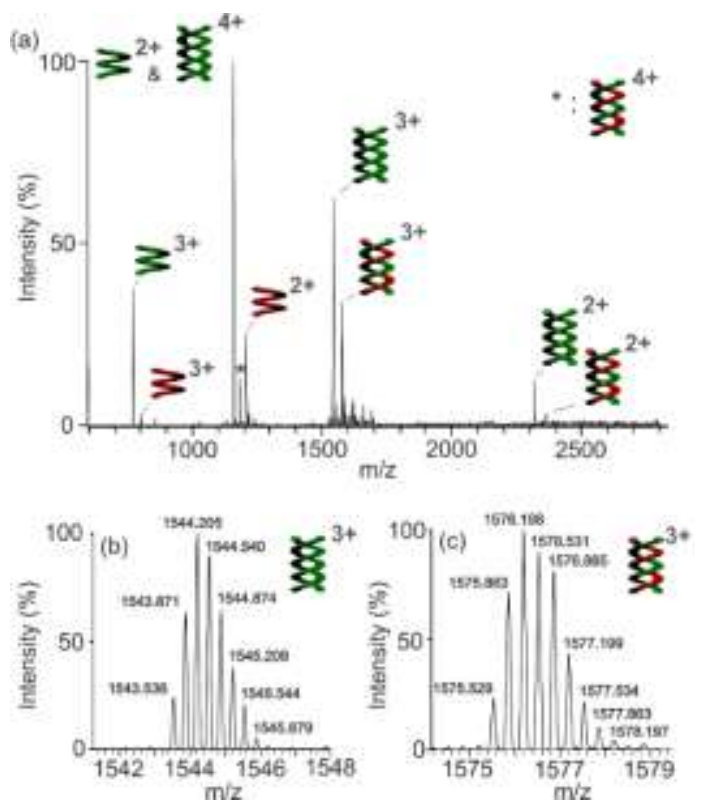


Figure 4. (a) Electrospray ionization mass spectrum (ESI-MS) of a solution of $20 \mu \mathrm{M}$ of oligomer 2 in water. The homo-duplex of 2 (green helices) and hetero-duplex of $\mathbf{2}-\mathrm{COCF}_{3}$ (red helices) are detected. Excerpts of the high resolution ESI mass spectrum of: (b) the homoduplex (2) 2 and (c) the heteroduplex $2 \cdot 2-\mathrm{COCF}_{3}$.

In summary, we have shown that water permits and greatly enhances aromatic oligoamide foldamers double helix formation. This family of double helices appear to be the only one that can form in virtually any solvent. SPS was shown to give rapid and easy access to oligomers comprised of different aromatic amide units and able to associate strongly in aqueous media. This may make water soluble $\left(\mathrm{Q}^{\mathrm{F}}\right)_{\mathrm{n}}$ oligomers become a convenient 'molecular glue' for the non-covalent conjugation of biomolecules or for use in biomaterials. In this respect, cross hybridization of distinct sequences could prove particularly useful. $^{3 \mathrm{~b}}$

\section{ASSOCIATED CONTENT}

\section{Supporting Information}

Experimental procedures and compound characterization data including NMR spectra and relevant HPLC chromatograms. This material is available free of charge via the Internet at http://pubs.acs.org.

\section{AUTHOR INFORMATION}

\section{Corresponding Author:}

* E-mail: y.ferrand@iecb.u-bordeaux.fr, i.huc@iecb.ubordeaux.fr

\section{Author Contributions}

All authors contributed to writing the manuscript and have given approval to its final version.

\section{ACKNOWLEDGMENT}

This work was supported by a CNRS PICS, by the Conseil Régional d'Aquitaine (predoctoral fellowship to Q.G.) and by the European Research Council under the European Union's Seventh Framework Programme (Grant Agreement No. ERC-2012-AdG320892, post-doctoral fellowship to S.J.D).

\section{REFERENCES}

(1) (a)Watson, J. D.; Crick F. H. C. Nature 1953, 171, 737-738. (b) Ramachandran, G. N. ; Kartha, G. Nature 1954, 174, 269-270. (c) Benedetti, E.; Di Blasio, B.; Pedone, C.; Lorenzi, G. P.; Tomasic, L.;
Gramlich, V. Nature 1979, 282, 630. (d) Wu, H-C. H.; Sarko, A. Carbohydrate Research 1977, 54, C3-C6.

(2) (a) Berl, V.; Huc, I.; Khoury, R.; Krische, M.J.; Lehn, J.-M. Nature 2000, 407, 720-723. (b) Baptiste, B.; Zhu, J.; Haldar, D.; Kauffmann, B.; Léger, J.-M.; Huc, I. Chem. Asian J. 2010, 5, 1364-1375. (c) Zhan, C.; Léger, J.-M.; Huc I. Angew. Chem. Int. Ed. 2006, 45, 46254628.

(3) (a) Gan, Q.; Bao, C.; Kauffmann, B.; Grélard, A.; Xiang, J.; Liu, S.; Huc, I.; Jiang, H. Angew. Chem. Int. Ed. 2008, 47, 1715-1718. (b) Gan, Q.; Li, F.; Li, G.; Kauffmann, B; Xiang, J.; Huc, I.; Jiang H. Chem. Commun. 2010, 46, 297-299. (c) Gan Q.; Shang, J.; Kauffmann, B.; Wang, Y.; Bie, F., Jiang H. Gan Q., Shang, J.; Kauffmann, B.; Wang, Y.; Bie, F., Jiang H. Tetrahedron 2012, 68, 4479-4484.

(4) Ferrand, Y.; Kendhale, A. M.; Garric, J.; Kauffmann, B.;Huc, I. Angew. Chem. Int. Ed. 2010, 49, 1778-1781.

(5) Goto, H.; Katagiri, H.; Furusho,Y.; Yashima, E. J. Am. Chem. Soc. 2006, 128, 7176-7178.

(6) (a) Amemiya, R.; Saito, N.; Yamaguchi, M. J. Org. Chem. 2008, 73, 7137-7144. (b) Sugiura, H.; Nigorikawa, Y.; Saiki, Y.; Nakamura, K.; Yamaguchi, M. J. Am. Chem. Soc. 2004, 126, 14858-14864.

(7) (a) Tanaka, Y.; Katagiri, H.; Furusho, Y.; Yashima, E. Angew Chem. Int. Ed. 2005, 44, 3867-3870. (b) Ito, H.; Furusho, Y.; Yashima, E. J. Am. Chem. Soc. 2008, 130, 14008-14015.

(8) (a) Li, J.; Wisner, J. A.; Jennings, M. C. Org. Lett. 2007, 9, $3267-$ 3269. (b) Mudraboyina, B. P.; Wisner, J. A. Chem. Eur. J. 2012, 18 , 14157-14164.

(9) (a) Goto, H.; Furusho, Y.; Yashima, E. J. Am. Chem. Soc. 2007, 129, 109-112; (b) Goto, H.; Furusho, Y.; Miwa, K.; Yashima, E. J. Am. Chem. Soc. 2009, 131, 4710-4719. (c) Ben, T.; Furusho, Y.; Goto, H.; Miwa, K.; Yashima, E. Org. Biomol. Chem. 2009, 7, 2509-2512.

(10) Gabriel, G. J.; Iverson B. L. J. Am. Chem. Soc. 2002, 124 15174-15175.

(11) Zhang, D.-W.; Zhao, X.; Hou, J.-L.; Li, Z.-T. Chem. Rev. 2012 $112,5271-5316$.

(12) (a) Gillies, E.; Deiss, F.; Staedel, C.; Schmitter, J.-M.; Huc, I. Angew. Chem. Int. Ed. 2007, 46, 4081-4084. (b) Iriondo-Alberdi, J., Laxmi-Reddy, K.; Bouguerne, B.; Staedel, C.; Huc, I. ChemBioChem 2010, 11, 1679-1685.

(13) Qi, T.; Maurizot, V.; Noguchi, H.; Charoenraks, T.; Kauffmann, B.; Takafuji, M.; Ihara, H.; Huc I. Chem. Commun. 2012, 48, 63376339.

(14) Bao, C.; Gan, Q.; Kauffmann, B.; Jiang, H.; Huc, I. Chem. Eur. J. 2009, 15, 11530-11536.

(15) (a) Baptiste, B.; Douat-Casassus, C.; Laxmi-Reddy, K.; Godde, F.; Huc, I. J. Org. Chem. 2010, 75, 7175-7185. (b) Wurtz, N. R.; Turner, J. M.; Baird, E. E.; Dervan, P. B. Org. Lett. 2001, 3, 12011203. (c) Puckett, J. W.; Green, J. T.; Dervan, P. B. Org. Lett. 2012, 14, 2774-2777. (d) Kolnig, H. M.; Abbel, R.; Schollmeyer, D.; Kilbinger, A. F. Org. Lett. 2006, 8, 1819-1822. (e) Murphy, N. S.; Prabhakaran, P.; Azzarito, V.; Plante, J. P.; Hardie, M. J.; Kilner, C. A.; Warriner, S. L.; Wilson, A. J. Chem. Eur. J. 2013, 19, 5546-5550. 